ARTICLE

Received 22 May 2014 | Accepted 12 Aug 2014 | Published 18 Sep $2014 \quad$ DOl: 10.1038/ncomms5963

\title{
Primate-specific miR-576-3p sets host defense signalling threshold
}

\author{
Melanie L. Yarbrough ${ }^{1}$, Ke Zhang ${ }^{1}$, Ramanavelan Sakthivel ${ }^{1}$, Christian V. Forst ${ }^{2}$, Bruce A. Posner ${ }^{3}$, Glen N. Barber ${ }^{4}$, \\ Michael A. White ${ }^{1} \&$ Beatriz M.A. Fontoura ${ }^{1}$
}

MicroRNAs (miRNAs) have been shown to regulate viral infection, but the miRNAs that target intracellular sensors and adaptors of innate immunity have not been fully uncovered. Here we conduct an miRNA mimic screen and validation with miRNA inhibitors in cells infected with vesicular stomatitis virus (VSV) to identify miRNAs that regulate viral-host interactions. We identify miR-576-3p as a robust regulator of infection by VSV and other RNA and DNA viruses. While an miR-576-3p mimic sensitizes cells to viral replication, inhibition of endogenous miR-576-3p prevents infection. miR-576-3p is induced by IRF3 concomitantly with interferon and targets STING, MAVS and TRAF3, which are critical factors for interferon expression. Interestingly, miR-576-3p and its binding sites are primate-specific and miR-576-3p levels are reduced in inflammatory diseases. These findings indicate that induction of miR-576-3p by IRF3 triggers a feedback mechanism to reduce interferon expression and set an antiviral response threshold to likely avoid excessive inflammation.

\footnotetext{
${ }^{1}$ Departments of Cell Biology, University of Texas Southwestern Medical Center, Dallas, Texas 75390-9039, USA. ${ }^{2}$ Department of Genetics and Genomic Sciences, Institute for Genomics and Multiscale Biology, Mount Sinai School of Medicine, New York, New York 10029-6574, USA. ${ }^{3}$ Department of Biochemistry, University of Texas Southwestern Medical Center, Dallas, Texas 75390-9039, USA. ${ }^{4}$ Department of Cell Biology and Sylvester Comprehensive Cancer Center, University of Miami Miller School of Medicine, Miami, Florida 33136, USA. Correspondence and requests for materials should be addressed to B.M.A.F. (email: beatriz.fontoura@utsouthwestern.edu).
} 
A lthough studies on the functions of host miRNAs during viral infection are still at early stages, increasing evidence suggests that miRNAs have a key role in the regulation of viral infection ${ }^{1}$. While viruses may utilize cellular miRNAs to promote their replication, miRNA expression can also facilitate the host antiviral response through interaction with viral RNA or by negatively regulating host factors required for viral proliferation. One example is miR-199a-3p, which has antiviral activity by regulating various pathways targeted by viruses such as the PI3K/AKT and ERK/MAPK pathways, oxidative stress signalling and prostaglandin synthesis ${ }^{2}$. Studies in vivo by Otsuka et al. ${ }^{3}$ demonstrated that mice harbouring a mutant Dicer allele were hypersusceptible to VSV infection, suggesting that host miRNAs contribute to host resistance to viral infection. VSV is a useful model virus for understanding viral infection processes as well as innate and adaptive immune responses. VSV is a negative sense RNA virus that replicates in the cytoplasm of most vertebrate and many invertebrate cell types ${ }^{4,5}$. During infection, viruses are susceptible to innate immune responses that induce type I interferon (IFN) and inflammatory cytokines. Upon cytoplasmic entry, viral RNA is recognized by a family of $\mathrm{DExD} / \mathrm{H}$-box helicases that include retinoic acid inducible gene 1 (RIG-I) and melanoma differentiation associated antigen 5 (refs 6,7). Upon interaction with viral RNA, RIG-I and melanoma differentiation associated antigen 5 associate with the adaptor protein MAVS (also known as IPS-1, VISA, or CARDIF) to facilitate activation of transcription factors that induce type I IFN signalling ${ }^{8-11}$. The efficient induction of antiviral response to intracellular viral RNA and also foreign DNA was shown to involve a central adaptor molecule STING (stimulator of IFN genes), an ER-associated protein that interacts with RIG-I and MAVS ${ }^{12,13}$. Recently, it was shown that foreign DNA such as that released by viruses in the cytoplasm is sensed by the enzyme cyclic GMP-AMP synthase (CGAS), which synthesizes cyclic-di-GMP-AMP molecules that are recognized by STING to induce IFN signalling ${ }^{14,15}$. Thus, type I IFN interferon production represents a major antiviral response pathway and STING is a key mediator of this response.

In this manuscript, we identify a novel primate-specific microRNA-driven feedback mechanism that prevents excessive innate immune signalling that can lead to abnormal inflammation. By using an unbiased genome-wide screening approach to search for miRNAs that regulate viral-host interactions, we identified miR-576-3p, which is induced by IRF3 and in turn downregulates key constituents of the IFN expression pathway, such as STING, MAVS and TRF3, to avert sustained immune signalling.

\section{Results and Discussion}

Identification of miRNAs that regulate viral infection. To identify novel miRNAs that are key regulators of viral infection, we analysed the impact of expressing a library of human miRNA mimics on the viability of cells infected with VSV (Fig. 1a). The miRNA mimics are synthetic RNA duplexes, which are functionally equivalent to mature miRNAs that have been processed by Dicer. Upon transfection into cells, the mimics lead to downregulation of target genes. The screen was conducted in human bronchial epithelial cells (HBECs) ${ }^{16}$, which represent a common site of viral infection. To determine that transfection of miRNA mimics themselves do not impact viral infection, a nontargeting miRNA mimic from Caenorhabditis elegans was used as a negative control and was included in each plate to normalize variations between plates. In addition, an siRNA to ubiquitin B (UBB), which leads to cell death, was used to monitor transfection efficiency and as a metric for total loss of cell viability. After transfection of miRNA mimics, cells were infected with a VSV strain that expresses GFP, so that the progression of infection could be monitored in live cells by fluorescence (Fig. 1b). Infection conditions were optimized so that control transfected cells exhibited approximately $50 \%$ cell death after $20 \mathrm{~h}$ of infection (Fig. 1c). This allowed for the identification of miRNAs that promote resistance or sensitivity to infection. A total of 810 mimics representing 723 unique miRNAs were analysed. Those mimics that resulted in toxicity upon transfection in the absence of VSV infection were excluded from further analysis (Fig. 1d and Supplementary Data 1).

miRNAs that impact viral infection were identified by their ability to increase or decrease cell viability in the presence of VSV infection compared with non-targeting mimic control. Cell viability ratios between infected and uninfected cells expressing the same miRNA were calculated and ranked from highest to lowest (Supplementary Data 2). Those miRNAs that increased or decreased cell viability were identified as resisters and sensitizers of VSV infection, respectively, and the top hits were selected. For confirmation, these miRNA hits were again transfected into HBECs followed by infection as in the primary screen (Fig. 1e) and their basal levels were measured by microRNA array (Supplementary Data 3). Eight miRNA resisters (Fig. 1e) and six miRNA sensitizers (Fig. 1f) were validated. miR-193b* was not confirmed as hit (Fig. 1f).

To determine if endogenous expression of the miRNAs could significantly impact viral infection, we transfected inhibitors of each of the confirmed hits from the miRNA mimic screen into HBECs and infected them with VSV. The inhibitors are singlestranded oligonucleotides that compete with a target mRNA for binding to endogenous mature miRNA. Transfection of inhibitors of miRNAs that resist VSV infection resulted in significantly decreased cell viability upon VSV infection for several hits, including miR-802, let-7e, let-7a, miR-98 and miR-125b (Fig. 1g). miR-98, let-7 miRNAs and miR-125b have been previously shown to regulate responses to microbial infection, serving as validation to our screen ${ }^{17-19}$. Inhibition of miRNAs that sensitized HBECs to viral infection resulted in increased cell viability during VSV infection for many of the top hits. This effect was observed with five miRNAs including miR-576-3p, miR-550, miR-138, miR-888 and miR-625 (Fig. $1 \mathrm{~h}$ ).

Since transfection of the mimic and inhibitor of miR-576-3p produced significant opposing effects on VSV infection (Fig. 1f,h) and because it more strongly promoted viral replication as compared with other novel hits, using the conditions shown in Fig. 2a, we investigated the unknown function of miR-576-3p in viral infection. Overexpression of miR-576-3p in VSV-GFP infected HBECs resulted in increased GFP production (Fig. 2a) and virus replication, as supernatants from these cells yielded an increase in viral titre compared with control transfected cells (Fig. 2b). Additional results of VSV replication in the presence of miR-576-3p mimic or inhibitor are shown in Supplementary Fig. 1. Indeed, enhanced viral replication (Fig. $2 b$ and Supplementary Fig. 1a) is corroborated by the increase in viral protein levels on overexpression of miR-576-3p (Fig. 2c; Supplementary Fig. 6). In contrast, inhibition of endogenous miR-576-3p function dramatically decreased viral protein levels (Fig. 2c; Supplementary Fig. 6) and viral replication (Supplementary Fig. 1b). To determine if regulation of the host response to viral infection by miR-576-3p is specific to VSV, we infected cells treated with miR-576-3p with influenza virus. As shown in Fig. 2d, miR-576-3p significantly sensitized HBECs to influenza virus infection and replication. In addition, HBECs overexpressing miR-576-3p had significantly decreased cell viability and produced more viral particles upon infection with vaccinia virus and Herpes simplex virus (HSV-1), which are DNA 
a

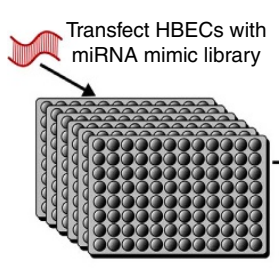

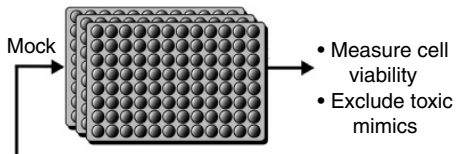

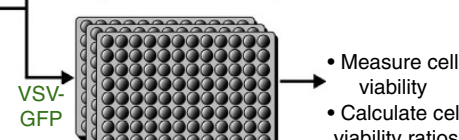

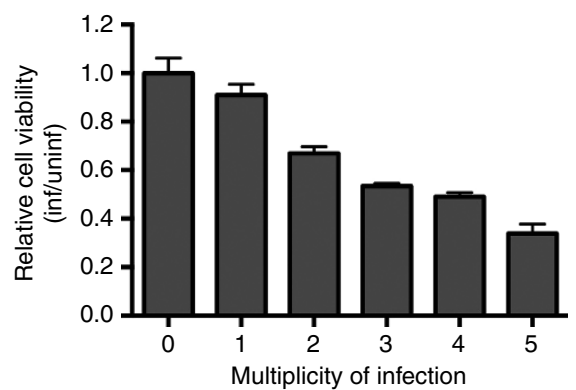

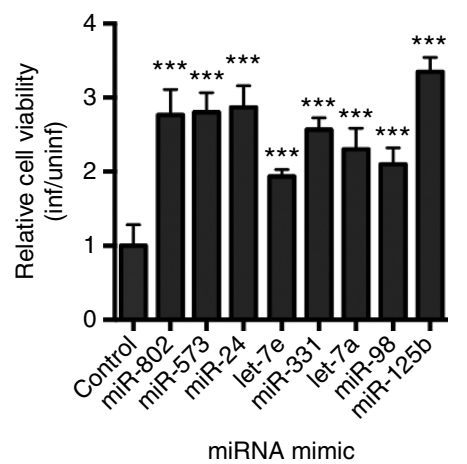

g

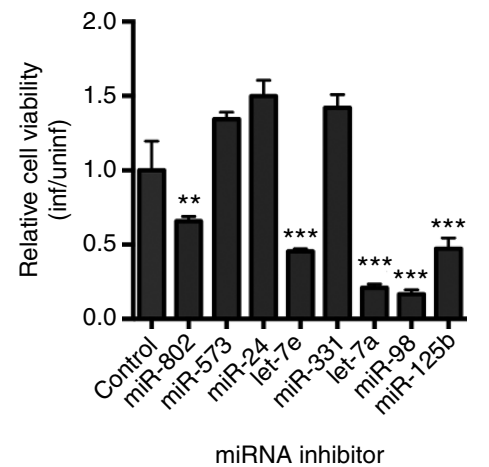

b

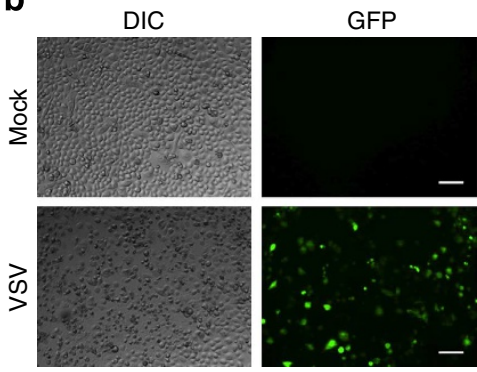

d

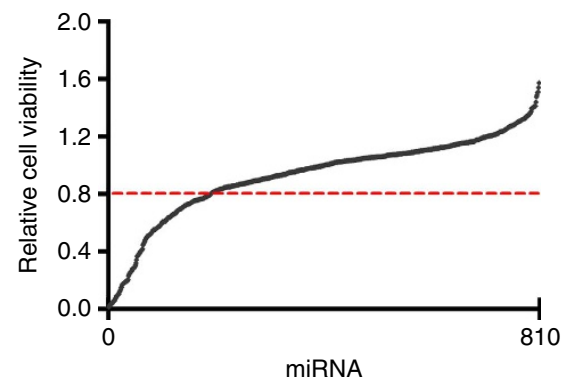

$\mathbf{f}$

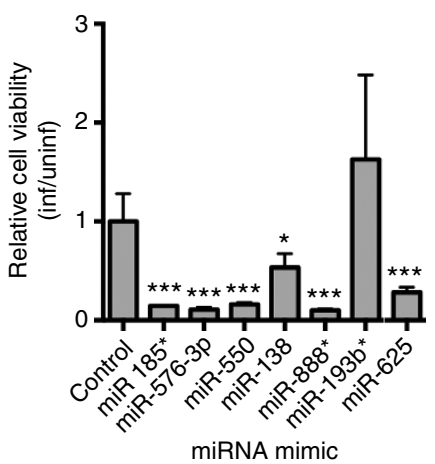

h

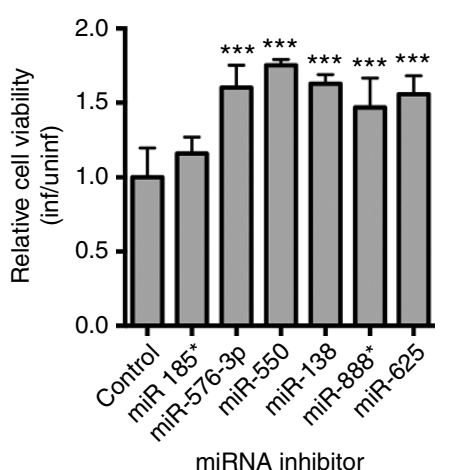

Figure 1 | Identification of host miRNAs that regulate VSV infection. (a) Design of high-throughput miRNA mimic screen. HBECs were reverse transfected with a library of miRNA mimics at a concentration of $50 \mathrm{nM}$. Control miRNA mimics and siRNAs to ubiquitin B (UBB) were included as negative and positive controls for cell death, respectively. After $72 \mathrm{~h}$ of transfection, cells were mock infected or infected with VSV-GFP at multiplicity of infection $(\mathrm{MOI})$ of 3 for $20 \mathrm{~h}$. Cytotoxicity was determined by measuring the ATP levels. Experimental values were normalized using two-point normalization to the medians of the miRNA mimic negative control and the UBB siRNA cytotoxicity control. (b) HBECs were infected with VSV-GFP and infection was monitored by fluorescence microscopy. Scale bar, $100 \mu \mathrm{M}$. (c) HBECs were infected (inf) with various multiplicities of infection (MOls) to determine the $\mathrm{MOI}$ resulting in $50 \%$ cell viability. Relative cell viability was determined by measuring the ATP levels. (d) Cell viability for each miRNA mimic-transfected well and infected as in a was measured. miRNA mimics were excluded as potential hits in the infected cell data set if viabilities in uninfected (uninf) cells were $<0.8$ (dotted red line). (e,f) The top resisters (e) and sensitizers (f) selected from the miRNA mimic screen were reverse transfected into HBECs and infected with VSV-GFP at an MOI of 3 for $20 \mathrm{~h}$. Cell viability was measured using Cell Titre Glo and was normalized to cells transfected with negative control miRNA mimic (Control). (g,h) HBECs were transfected with inhibitors to the indicated miRNAs for $72 \mathrm{~h}$. Infection and measurements of cell viability were performed as in $\mathbf{e}$ and normalized to cells transfected with negative control miRNA inhibitor (control). Data are representative of at least three independent experiments and unpaired two-tailed $t$-test was used. Error bars represent s.d. ${ }^{\star} P<0.05,{ }^{\star \star} P<0.01,{ }^{\star \star \star} P<0.001$. 
a
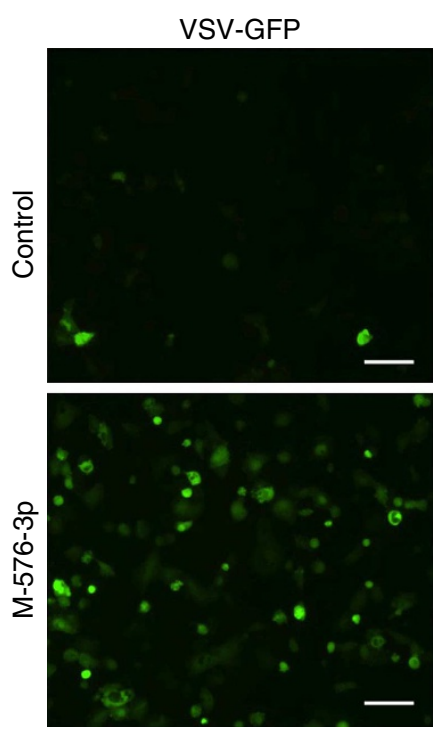

d
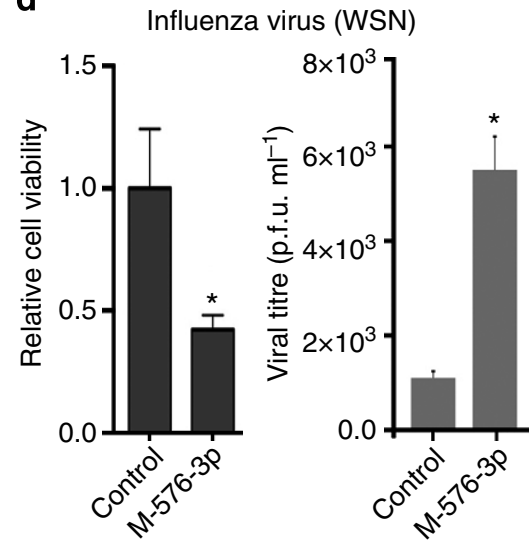

b

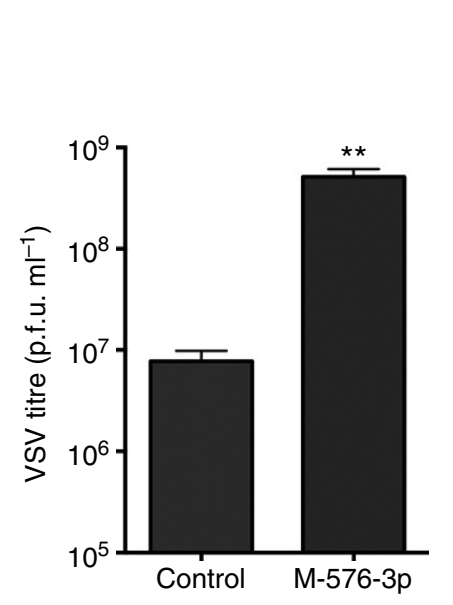

C

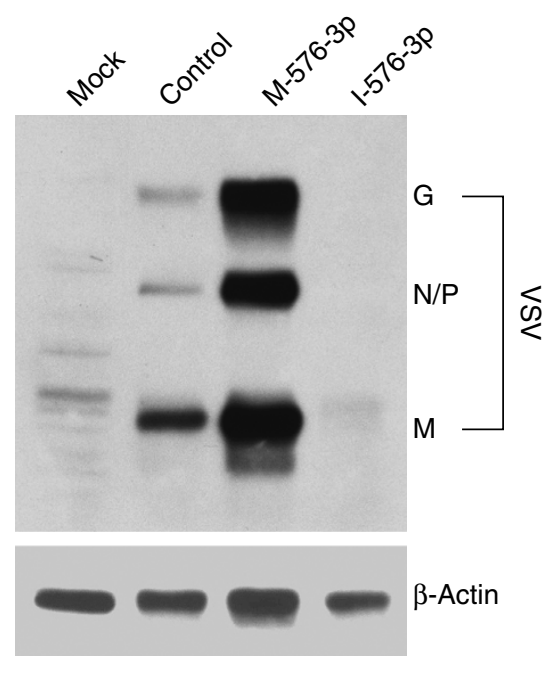

$\mathbf{f}$

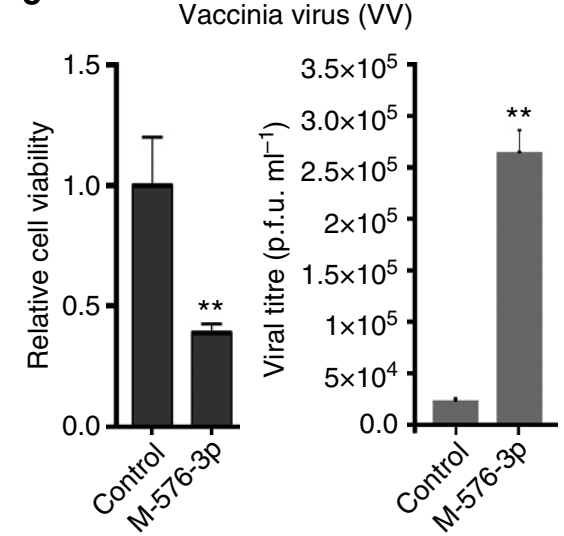

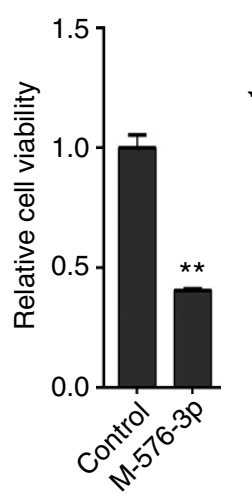

Herpes simplex virus (HSV-1)

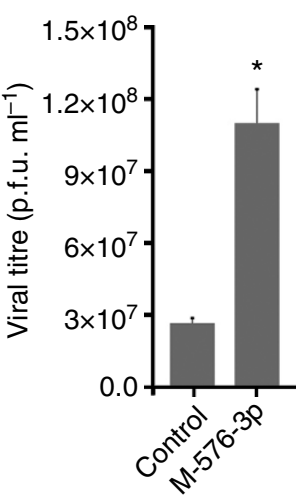

Figure 2 | miR-576-3p is a broad regulator of viral infection. (a) HBECs were transfected with miRNA mimic control, miR-576-3p mimic (M-576-3p) and infected with VSV-GFP at an MOI of 1 for $20 \mathrm{~h}$. VSV-GFP production was monitored by fluorescence microscopy. Scale bar, $100 \mu \mathrm{M}$. (b) Samples of supernatant from infected cells in a were collected and viral titres were measured by plaque assays. (c) HBECs were transfected with miRNA mimic control (Control), miR-576-3p mimic (M-576-3p) or miR-576-3p inhibitor (I-576-3p) and infected with VSV-GFP at an MOI of 1 for $20 \mathrm{~h}$. Cell lysates were collected and VSV protein levels were measured by western blot with anti-VSV antibodies. (d) HBECs were transfected with control or miR-576-3p mimic (M-576-3p). After $72 \mathrm{~h}$ of transfection, cells were infected with the WSN strain of influenza virus at MOI of 5 for $18 \mathrm{~h}$. Cell viability and viral replication were measured by Cell Titre Glo and plaque assays, respectively. (e) HBECs were transfected as in $\mathbf{d}$ and infected with vaccinia virus at MOI of 3 for $18 \mathrm{~h}$ and cell viability was measured as in $\mathbf{d}$. Viral titre was measured by plaque assay. (f) HBECs were transfected as in $\mathbf{d}$ and infected with HSV-1 at MOI of 0.1 for $96 \mathrm{~h}$. Cell viability was measured as in $\mathbf{d}$ and viral titre was determined by plaque assay. Data are representative of at least three independent experiments and unpaired two-tailed $t$-test was used. Error bars represent s.d. ${ }^{\star} P<0.05,{ }^{\star \star} P<0.01$. p.f.u., plaque forming unit.

viruses, compared with cells transfected with control miRNA (Fig. 2e,f). Altogether, our findings indicate that miR-576-3p sensitizes cell to viral infection and that it is a broad regulator of infections by both RNA and DNA viruses.

miR-576-3p targets constituents of innate immune signalling. To identify mRNA targets of miR-576-3p, we performed gene expression profiling with RNA obtained from HBECs transfected with a mimic or inhibitor of miR-576-3p, and compared individual mRNA levels with control transfected cells (Supplementary Data 4). Gene set enrichment analysis (GSEA) of these mimic and inhibitor conditions identified cellular pathways that are potentially targeted by miR-576-3p. Among the top pathways showing the best enrichment scores was cytokine signalling, which included mRNAs that encode constituents of the RIG-I signalling pathway. After leading edge analysis, mRNAs whose expression decreased upon miR-576-3p overexpression and increased upon inhibition of the endogenous miR-576-3p were selected as potential targets of miR-576-3p. A key response network regulated by miR-576-3p was then generated. Next, all mRNAs within the response network were analysed for the presence of miR-576-3p seed regions using the TargetScan prediction program ${ }^{20}$. This analysis showed that 18 of the 94 mRNAs in the response network contained potential miR-576-3p target sites (Fig. 3a).

To assess whether differences in the levels of the selected mRNAs are a result of direct targeting by miR-576-3p, the $3^{\prime}$ untranslated region (UTR) of each mRNA containing predicted target sites for miR-576-3p was cloned downstream of a luciferase reporter gene and co-transfected with miR-576-3p or control mimic into $293 \mathrm{~T}$ cells. We found that miR-576-3p significantly decreased luciferase expression in 13 of the 18 UTRs identified in our GSEA (Fig. 3b). Furthermore, deletion of the region 
a
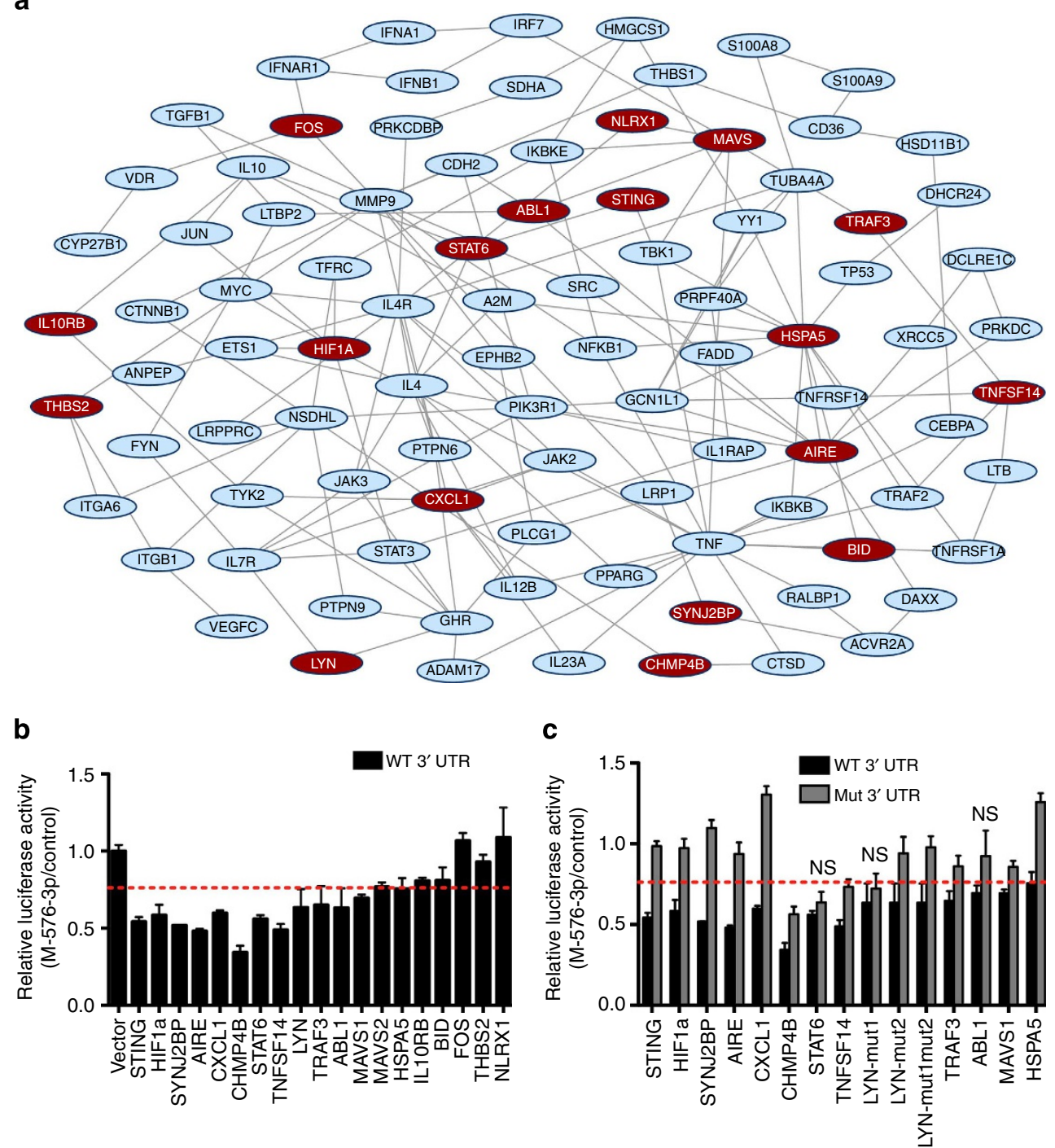

Figure 3 | miR-576-3p targets key players of immune response pathways. (a) Gene expression profiling was performed with RNA obtained from HBECs transfected in triplicate with miRNA mimic control or miR-576-3p mimic, and miRNA inhibitor control or miR-576-3p inhibitor. GSEA was performed for both conditions. Response network illustrates the regulation of closely connected host immune signalling pathways in response to treatment of HBECs with miR-576-3p mimic or inhibitor. Nodes (proteins) in the network are connected by an edge on the basis of physical and/or functional interactions. Red colour nodes denote mRNAs containing miR-576-3p-specific seed regions $(P<0.0005)$. The parameters of network analysis are $k=1$ and $I=5$. (b) Dual-luciferase assays were performed on HEK293T cells transfected with control or miR-576-3p mimic (M-576-3p) and luciferase constructs encoding wild-type $3^{\prime}$-UTR of genes predicted to be regulated by miR-576-3p inserted downstream of luciferase. Empty vector was transfected as a negative control. Luciferase expression was normalized to a Renilla luciferase expression and relative luciferase activity of miR-576-3p mimic (M-576-3p) transfected wells was compared with mimic control luciferase levels. Dashed red line indicates the threshold (75\% of luciferase activity). (c) Dualluciferase assays of HEK293T cells transfected with control or miR-567-3p mimic (M-576-3p) as in $\mathbf{b}$ and luciferase constructs encoding wild-type (WT) or mutated (mut) $3^{\prime}$-UTR (with deletion of miR-576-3p seed sequence) of genes predicted to be targets in $\mathbf{a}$. Data were normalized as in $\mathbf{b}$ and are representative of at least three independent experiments. NS indicates mutants that are not significantly different from wild-type $3^{\prime}$-UTRs. Unpaired two-tailed $t$-test was used and error bars represent s.d.

complementary to the miR-576-3p seed sequence in these UTRs significantly eliminated the inhibitory effect of miR-576-3p on luciferase expression in all but three of the mutant $3^{\prime}$-UTRs (Fig. 3c), indicating that these genes are direct targets of miR-576-3p. Among these targets is STING (Fig. 3b,c), which is critical for type I IFN expression and antiviral signalling ${ }^{12,13}$.

Examination of STING at the mRNA and protein levels revealed that expression of STING was reduced in mock and VSV infected cells transfected with miR-576-3p mimic, and STING protein levels increased in the presence of miR-576-3p inhibitor as compared with control (Fig. 4a-c; Supplementary Fig. 6). In addition, MAVS and TRAF3 mRNAs, which together with
STING are constituents of the pathway that leads to IFN expression and antiviral signalling, also appear to be targets of miR-576-3p (Fig. 3b,c). In fact, MAVS and TRAF3 protein levels increased in the presence of miR-576-3p inhibitor during infection (Supplementary Figs 2 and 6). These data thus indicate that miR-576-3p regulates antiviral signalling by targeting key factors within the pathogen recognition and host response pathway.

miR-576-3p is induced by IRF3. We then investigated whether expression of miR-576-3p was regulated upon viral genome 
a

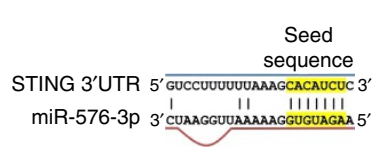

f

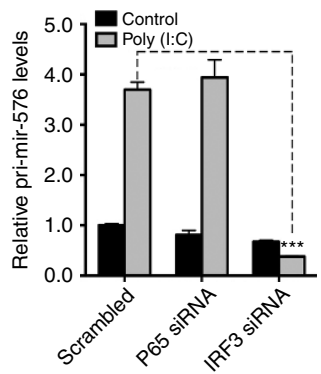

b

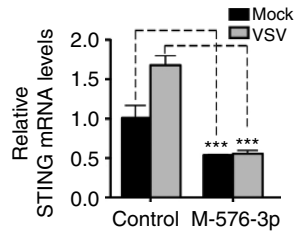

C

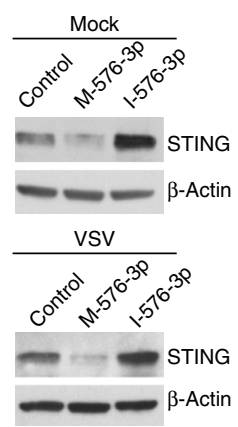

d

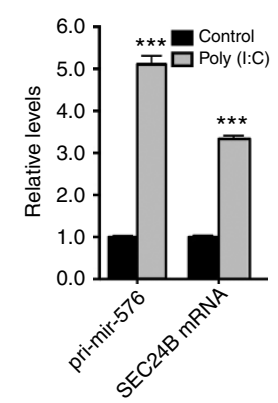

e

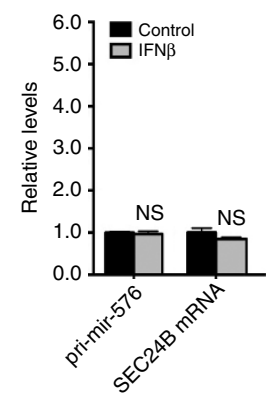

h

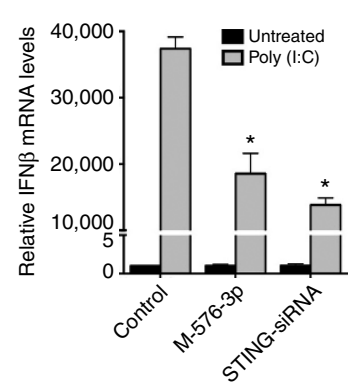

i

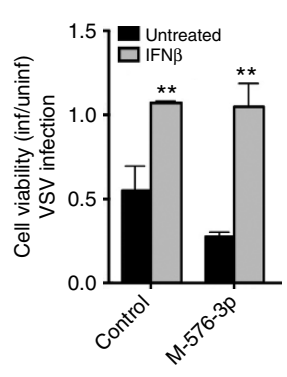

j

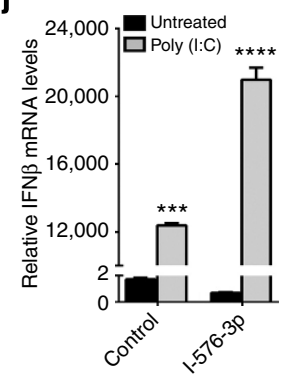

Figure 4 I miR-576-3p regulates Type I IFN signalling. (a) Schematic showing the region of the $3^{\prime}$-UTR of STING that is recognized by the miR-576-3p seed sequence. (b) HBECs were transfected with control or miR-576-3p (M-576-3p) mimic for $72 \mathrm{~h}$. Cells were then mock infected or infected with VSV-GFP at an MOI of 3 for $3 \mathrm{~h}$. Total RNA was collected from cells and STING mRNA levels were determined by qPCR and normalized to levels of $\beta$-actin. (c) HBECs were transfected with miRNA mimic (M-576-3p) or inhibitor (I-576-3p) as in $\mathbf{b}$ and mock infected or infected with VSV-GFP at MOI of 0.1 for $18 \mathrm{~h}$. Cell lysates were collected and subjected to western blot analysis with anti-STING antibodies. $\beta$-Actin serves as loading control. (d,e) HBECs were transfected with $1 \mu \mathrm{g} \mathrm{ml}^{-1}$ of poly (I:C) for $6 \mathrm{~h}(\mathbf{d})$ or treated with $100 \mathrm{U} \mathrm{ml}{ }^{-1}$ of IFN $\beta$ for $18 \mathrm{~h}(\mathbf{e})$ and levels of pri-mir-576 and SEC24B mRNA were analysed by qPCR. (f,g) HBECs were transfected with poly (I:C) as in d after siRNA knockdown of NFkB (P65) or IRF3. Relative pri-mir-576 (f) or SEC24B mRNA (g) levels were measured by qPCR. (h) HBECs-expressing control or miR-576-3p mimic (M-576-3p) or an siRNA targeting STING were transfected with poly (I:C) and levels of IFN $\beta$ mRNA were measured by qPCR. (i) HBECs were transfected with control or miR-576-3p mimic (M-576-3p) and pretreated with $100 \mathrm{U} \mathrm{ml}^{-1}$ of IFN $\beta$ before VSV infection. Cell viability was determined by measuring ATP levels of mock or infected cells. (j) HBECs-expressing control or miR-576-3p inhibitor (I-576-3p) were transfected with poly (I:C) and levels of IFN $\beta$ were measured by qPCR. Data are representative of three independent experiments. Unpaired two-tailed $t$-test was used and error bars represent s.d. ${ }^{\star} P<0.05,{ }^{\star \star} P<0.01,{ }^{\star \star \star} P<0.001,{ }^{\star \star \star \star} P<0.0001$.

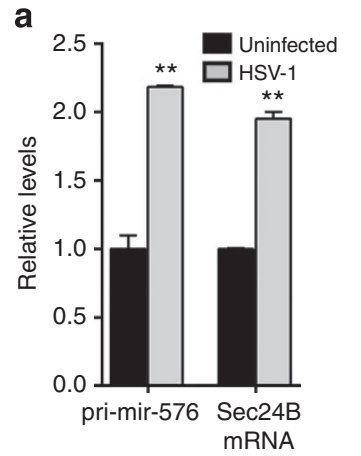

b

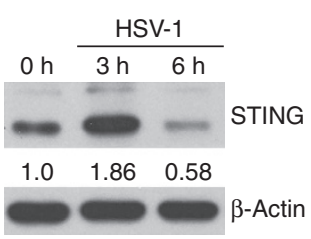

C

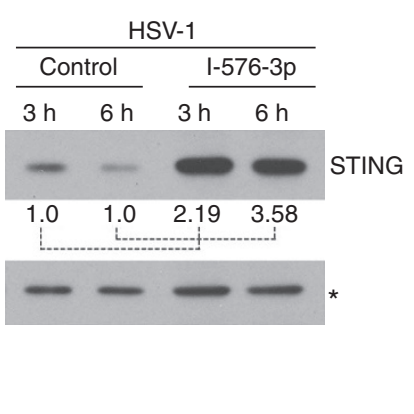

Figure 5 | Pri-mir-576 and STING protein levels are regulated during HSV-1 infection. (a) HBECs were mock infected or infected with HSV-1 at $\mathrm{MOI}$ of 10 for $3 \mathrm{~h}$ and levels of pri-mir-576 and SEC24B were measured by qPCR. Unpaired two-tailed $t$-test was used and error bars represent s.d. ${ }^{\star \star} P<0.01$. (b) HBECs were mock infected or infected with HSV-1 at MOI of 10 and STING proteins levels were measured by western blot. (c) HBECs were transfected with miRNA control or miR-576-3p inhibitor (I-576-3p) and infected with HSV-1 at MOI of 10. Cell lysates were collected at the indicated time points and subjected to western blot analysis. Relative STING levels to controls were quantified using ImageJ. Control values at $3 \mathrm{~h}$ and $6 \mathrm{~h}$ were set to 1 for comparison with samples treated with 1-576-3p. * denotes cross-reacting band that serves as loading control.

recognition and induction of antiviral response. The primary transcript of mir-576 is encoded within an intron of the Sec24B gene. Thus, Sec24B mRNA and mir-576 primary miRNA levels were measured on HBECs transfected with a low molecular weight form of poly (I:C), a synthetic RNA that mimics the effect of viral infection by activating the RIG-I pathway and type I IFN expression $^{21}$, or treatment with IFN $\beta$. Both Sec24B and pri-mir576 RNA levels were upregulated upon transfection of poly (I:C) when compared with control transfected cells (Fig. 4d), whereas cells treated with IFN $\beta$ exhibited no increase in either Sec24B mRNA or pri-mir-576 (Fig. 4e). These findings indicate that primir-576 is regulated by the RIG-I pathway but not by type I IFN. 

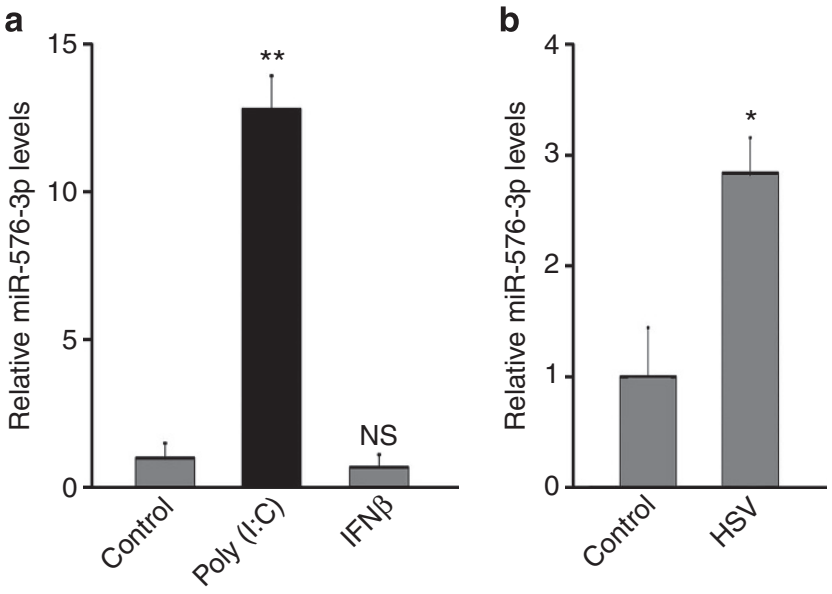

Figure 6 | miR-576-3p is upregulated by poly (I:C) and viral infection but not by interferon. (a) HBECs were transfected with $1 \mu \mathrm{g} \mathrm{ml} \mathrm{l}^{-1}$ of poly (I:C) for $6 \mathrm{~h}$ or treated with $100 \mathrm{U} \mathrm{ml}^{-1}$ of IFN $\beta$ for $18 \mathrm{~h}$ and levels of mature miR-576-3p were determined by qPCR. (b) HBECs were mock infected or infected with HSV-1 at MOI of 10 for $3 \mathrm{~h}$ and levels of miR-576-3p were measured by qPCR. Unpaired two-tailed $t$-test was used and error bars represent s.d. ${ }^{\star} P<0.05$, ${ }^{\star \star} P<0.01$. NS, nonsignificant with respect to control.

Since the RIG-I pathway induces cytokine production via IRF3 or $\mathrm{NF \kappa B}$, we tested both arms of the pathway to determine the mediator of pri-mir-576 expression. The increase in pri-mir-576 and Sec24B mRNA expression on transfection of HBECs with poly (I:C) was abrogated upon siRNA knockdown of IRF3 but not the p65 component of the NFKB heterodimer (Fig. 4f,g; Supplementary Fig. 3; Supplementary Fig. 4; Supplementary Fig. 6), indicating that mir-576 expression was specifically upregulated by IRF3. These results suggest that cells may upregulate miR-576-3p in response to invading viruses as a feedback mechanism to fine-tune IFN expression and the innate immune response to avoid excessive inflammation, which could damage host tissues.

As miR-576-3p targets STING and other important antiviral signalling proteins in the RIG-I pathway, we then assessed the effect of miR-576-3p on the production of type I IFN. Upon transfection of poly (I:C), levels of IFN- $\beta$ were significantly decreased in HBECs overexpressing miR-576-3p or with siRNA knockdown of STING when compared with control (Fig. 4h). Altogether, these results indicate that miR-576-3p directly regulates the production of type I IFN via STING and the RIG-I pathway. In support of this finding, pretreatment of HBECs transfected with either miR-576-3p or mimic control with IFN $\beta$ restored the viability of cells infected with VSV (Fig. 4i). Furthermore, HBECs transfected with miR-576-3p inhibitor increased IFN $\beta$ production in the presence of poly (I:C) (Fig. 4j), further corroborating the role of miR-576-3p as a regulator of IFN $\beta$ expression.

Regulation of pri-mir-576 and STING proteins levels were also detected during infection with both RNA and DNA viruses. In the case of VSV infection, pri-mir-576 levels were upregulated and then downregulated over time (Supplementary Fig. 5a) and STING proteins levels followed the opposite pattern, as expected for a target of miR-576-3p (Supplementary Figs $5 b$ and 6). Expression of miR-576-3p inhibitor then increased STING protein levels during VSV infection (Supplementary Figs $5 \mathrm{c}$ and 6), indicating a role for miR-576-3p on the regulation of STING levels during infection. HSV-1 infection also regulated pri-mir576 and Sec24B mRNA levels (Fig. 5a) and STING protein levels (Fig. 5b; Supplementary Fig. 6). Cells transfected with miR-576-

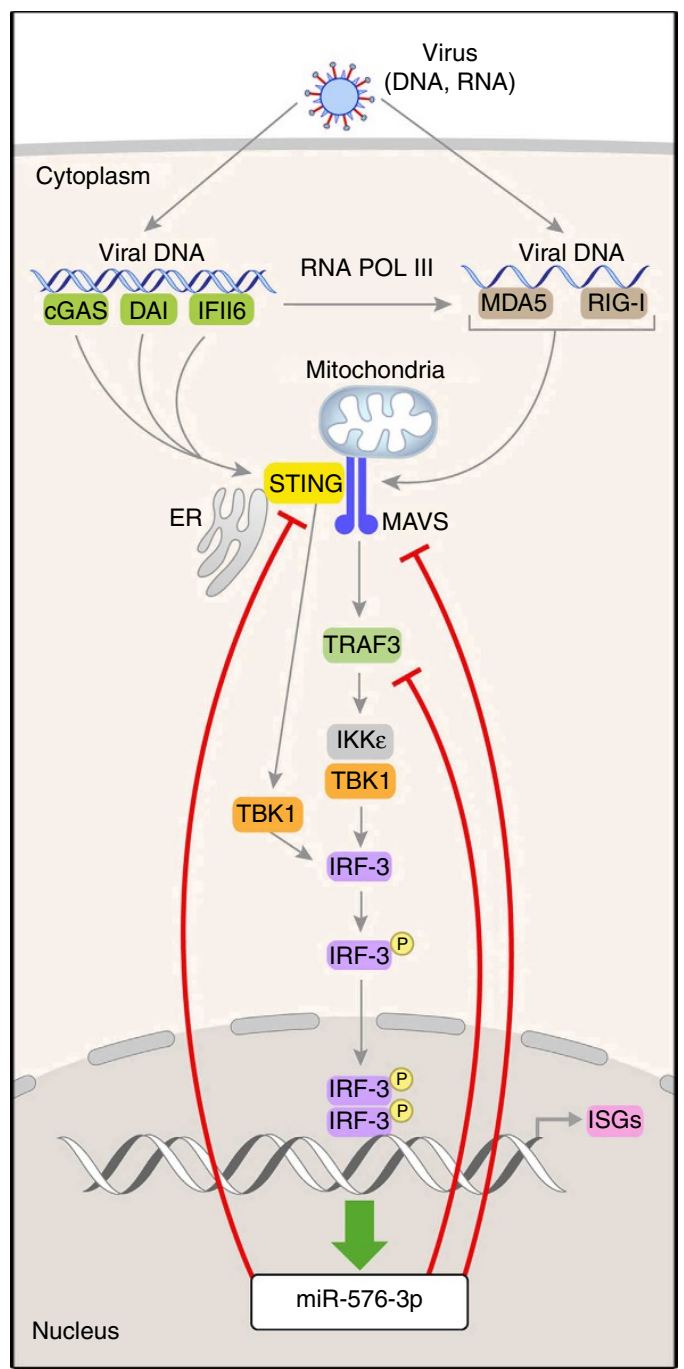

b

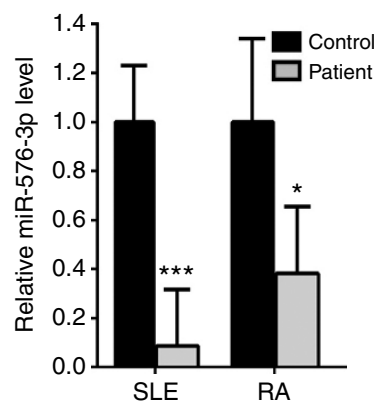

Figure 7 | miR-576-3p regulation in viral infection and other diseases. (a) Model illustrates regulation of IFN expression by miR-576-3p as a feedback mechanism. Targets of miR-576-3p are indicated by inhibitory red lines. (b) Public available microRNA array data sets (GSE37425 and GSE37426; ref. 23) of synovial or renal tissues from rheumatoid arthritis (RA) or systemic lupus erythematosus (SLE) patients, respectively, along with controls were obtained from the GEO database ${ }^{22}$. Normalized expression values of miR-576-3p from each sample were used to calculate the relative levels and $P$ values of miR-576-3p for SLE or RA patients as compared with controls. Unpaired two-tailed $t$-test was used and error bars represent s.d. ${ }^{\star} P<0.05,{ }^{\star \star \star} P<0.001$. 
pri-mir-576

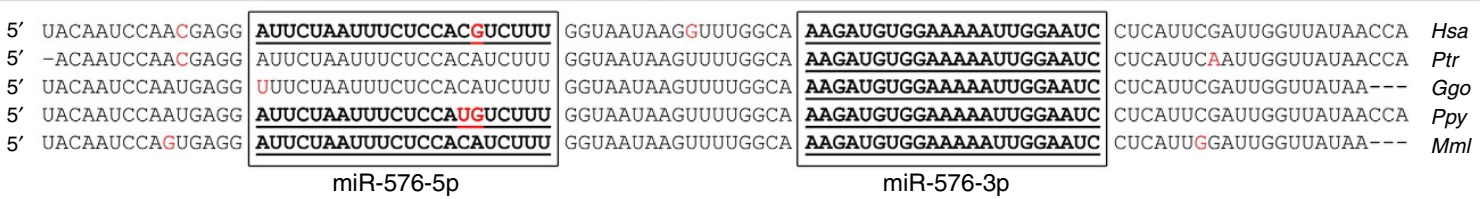

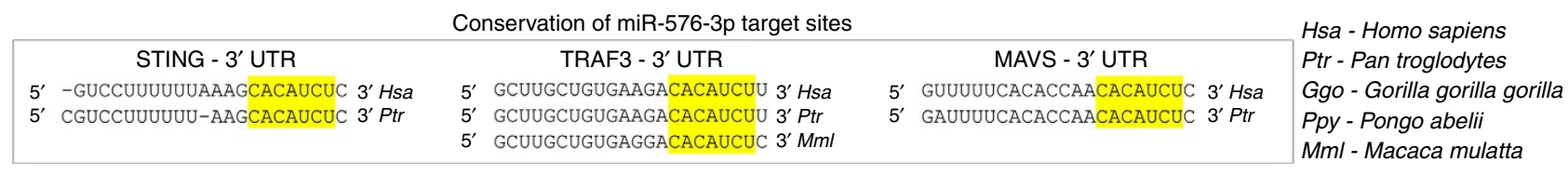

Figure 8 | miR-576-3p is primate-specific. Top: sequence alignment of pri-mir-576 from various species where it is present. miR-576-5p and miR-576-3p sequences are shown in boxes. Detected mature miR-576-5p and miR-576-3p are underlined. Red letters depict mismatched nucleotides between species. Bottom: multiple sequence alignments of representative miR-576-3p target sites in the indicated $3^{\prime}-U T R$. The miRNA seed region is highlighted in yellow.

$3 p$ inhibitor and infected with HSV-1 showed increased STING protein levels, indicating again a role for miR-576-3p in regulating STING levels during $\mathrm{HSV}-1$ infection as well (Fig. 5c; Supplementary Fig. 6). Like pri-mir-576, miR-576-3p levels were upregulated upon poly (I:C) treatment or by viral infection, but not by interferon (Fig. 6). Taken together, miR576-3p can target and downregulate STING during infection by both RNA and DNA viruses. A model could be proposed in which upregulation of miR-576-3p by IRF3, concomitantly with IFN, would downregulate STING, MAVS, and TRAF3 as a feedback mechanism to prevent continuous signalling and exacerbated inflammation (Fig. 7a). In fact, miR-576-3p levels are drastically downregulated in patients with systemic lupus erythematosus and rheumatoid arthritis (Fig. $7 \mathrm{~b})^{22,23}$, supporting the model of an antiinflammatory function for miR-576-3p. Another study proposed an miRNA-driven feedback mechanism for miR-146, which targets TNF receptor-associated factor 6 and IL-1 receptor-associated kinase 1 genes and thereby regulates Toll-like receptor and cytokine signalling ${ }^{24}$.

miR-576-3p is primate-specific. Interestingly, pri-mir-576 is only present in higher primates. Furthermore, only mature miR-576-3p, but not miR-576-5p, is detected and is identical in higher primates (Fig. 8). Consequently, miR-576-3p target sites are only found at the $3^{\prime}$-UTR of mRNAs from higher primates. STING, MAVS and TRAF3 are present in lower organisms but their mRNAs lack miR-576-3p target sites as opposed to their mRNAs from higher primates (Fig. 8). Altogether, these findings define a new layer of complexity that appeared late in evolution that may provide higher primates with a sophisticated mechanism to fine-tune the immune response and to efficiently trigger an antiinflammatory feedback mechanism.

\footnotetext{
Methods

Cell culture and reagents. Immortalized HBECs were obtained from J. Minna (UT Southwestern Medical Centre) and were cultured in keratinocyte serum-free media (Invitrogen) supplemented with EGF and bovine pituitary extract. Human embryonic kidney (HEK) 293T cells were cultured in DMEM (Invitrogen) with $10 \%$ fetal bovine serum (Atlas Biologicals). The following antibodies were used for immunoblot analysis according to the manufacturer's instructions: anti-STING rabbit polyclonal (Cell Signaling Technology), anti-MAVS rabbit polyclonal (Enzo Life Sciences), anti-TRAF3 rabbit polyclonal (Cell Signaling Technology), anti-P65 rabbit polyclonal (Santa Cruz), anti-IRF3 rabbit polyclonal (Santa Cruz), and anti- $\beta$-actin mouse monoclonal antibodies (Sigma). Antibodies against VSV proteins were used as previously reported ${ }^{25}$. The miRIDIAN human microRNA mimic library (miRBase version 10.1) was obtained from Dharmacon, Thermo. A total of 810 mimics were screened, which represented 723 unique mature miRNAs. Non-targeting control C. elegans miRNAs and siRNAs were purchased from Dharmacon. Recombinant human IFN $\beta$ was obtained from R\&D systems.
}

miRNA mimic screening and transfection. The library of miRNA mimics was re-suspended and arrayed in 96-well plates. HBECs were reverse transfected in replicates of six with miRNA mimics at a concentration of $50 \mathrm{nM}$ using Dharmafect 3 (Dharmacon), according to the manufacturer's instructions. Cells were added to the transfection mix at a density of $1 \times 10^{4}$ cells per well. Control miRNA mimics and siRNAs to UBB were included on each plate as negative and positive controls for cell death, respectively. After $72 \mathrm{~h}$ of transfection, cells were infected with VSV-GFP (Indiana strain) at a multiplicity of infection (MOI) of 3 for $20 \mathrm{~h}$. Cytotoxicity was determined by measuring ATP levels using the CellTiter-Glo luminescent assay (Promega), according to manufacturer's instructions. The Genedata Screener software (version 10.1, GeneData, Inc. Basel, Switzerland) was used to process and analyse the primary screening data. Experimental results were obtained from EnVision multi-label plate reader and processed using the Assay Analyzer module of the Genedata Screener Suite. In each 96-well assay plate, the miRIDIAN human microRNA mimic library (miRBase version 10.1) is located in columns $2-11$, leaving columns 1 and 12 open for controls. For each plate, the raw data values for all wells were normalized using the positive and neutral controls on each plate according to the following equation:

$$
\text { Normalized value }=\frac{\text { Raw data }- \text { Median MC1Ctrl }}{\text { Median PosCtrl }- \text { Median MC1Ctrl }}
$$

In this equation, $\mathrm{MC1}$ represents a $C$. elegans microRNA that does not target human mRNAs (neutral control wells B1, E1, B12, E12) and UBB served as the positive control (positive control wells D1, G1, D12, G12). Wells A1, C1, F1, H1, $\mathrm{A} 12, \mathrm{C} 12, \mathrm{~F} 12$ and $\mathrm{H} 12$ were used as untreated controls. Normalized well values were then tested for positional effects using a proprietary pattern detection algorithm in the Assay Analyzer software (See GeneData user documentation and reference ${ }^{26}$ ). No reproducible positional effects were identified. The median of the normalized values for the three replicates for each miRNA was calculated for both infected and uninfected cells. Hits that sensitized cells to virus infection were selected using the following criteria: $0.8<$ median uninfected cells $<1.2$ and median infected cells $<0.4$. miRNAs that protected cells from viral infection met the following criteria: $0.8<$ median uninfected cells $<1.2$ and median infected cells $>1.6$.

Cell viability ratios between virus-infected and -uninfected wells were calculated and miRNAs were ranked by their ability to increase cell viability upon infection. The top eight miRNAs with the highest increases in cell viability were selected as resisters of viral infection. Six miRNAs with the highest decreases in cell viability were selected as sensitizers to viral infection. These miRNAs were selected and retested in the assay using the conditions of the screen. Relative cell viabilities were normalized to miRNA mimic control transfected wells and cell viability ratios were calculated between infected and uninfected wells. Basal levels of these miRNAs were determined by microRNA array analysis and showed significant levels.

microRNA array analysis. Total RNA was harvested in TRIzol, according to the manufacturer's protocol. Two hundred nanograms of total RNA from three different HBECs samples were subjected to microRNA array analysis using the Human Illumina's MicroRNA Expression Profiling platform (humanMI_V2). miRNA levels were detected and normalized to negative controls, which are constituted of oligos targeting random sequences that are not present in the human genome. $P$ values were calculated using unpaired two-tailed $t$-test.

miRNA inhibitor transfection. The endogenous activity of miRNAs that were selected as resisters and sensitizers to VSV infection was blocked by the transfection of miRNA inhibitors (Dharmacon) for the indicated miRNA. Inhibitors were reverse transfected in HBECs at a concentration of $75 \mathrm{nM}$ using Dharmafect 3. After $72 \mathrm{~h}$, cells were processed as indicated in the figure legends. 
miR-576-3p target identification by microarray. For identification of genes regulated by miR-576-3p, HBECs were reverse transfected in triplicate with miR-576-3p mimic ( $50 \mathrm{nM})$ or inhibitor $(75 \mathrm{nM})$ and compared with cells transfected with miRNA mimic or inhibitor negative controls. After $72 \mathrm{~h}$ of transfection, total RNA was harvested in TRIzol, according to the manufacturer's protocol. Five hundred nanograms of total RNA was labelled using the Illumina TotalPrep kit (Ambion) and the biotin-labelled cRNA was hybridized to an Illumina HumanHT-12 v4 Expression BeadChip array according to the manufacturer's protocol.

GSEA of microarray data. Using raw data from the Illumina microarray, the expression values of genes from multiple oligonucleotide probes were averaged and the data file was formatted as required by the GSEA software ${ }^{27}$. GSEA was performed on microarray profile of mimic control against miR-576-3p mimic using standard mode with gene set permutations. Curated C2/CP canonical pathways Molecular Signatures Database (MSigDB, Broad Institute) v3.0 with 880 gene sets (pathways) has been used as reference in GSEA. GSEA was then repeated for inhibitor control against miR-576-3p inhibitor microarray data. Common gene sets between those genes downregulated by miR-576-3p mimic and upregulated by miR-576-3p inhibitor were selected and subjected to leading edge GSEA. The responding genes from leading edge analysis were further filtered for genes that were both downregulated by miR-576-3p mimic and upregulated by miR-576-3p inhibitor, resulting in 24 responding genes. These 24 responding genes, which were potential targets of miR-576-3p, were selected as seed nodes for protein network analysis, as previously reported ${ }^{28}$, using signal-to-noise ratios ranked on the basis of GSEA. The response network contained 94 proteins and 164 interactions regulated by miR-576-3p. mRNAs encoding the 94 proteins were checked for miR-576-3p seed region using TargetScanHuman version 6.2 and 18 mRNAs were found to have seed regions for miR-576-3p. To identify the biological theme of the response network targeted by miR-576-3p, the 94 genes were subjected to overlap analysis using MSigDB gene sets (http://www.broadinstitute.org/gsea/msigdb/ annotate.jsp) and showed enrichment for immune response pathways.

RNA isolation and qPCR. Total RNA was isolated using TRIzol reagent (Invitrogen) as recommended by the manufacturer. Reverse transcription of $1 \mu \mathrm{g}$ of total RNA was performed using Superscript II reverse transcriptase (Invitrogen) and $250 \mathrm{ng}$ of random hexamer primer, according to the manufacturer's instructions. Real-time PCR was performed using TaqMan Universal PCR master mix (Applied Biosystems) and the TaqMan Pri-miRNA Assay (hsa-mir-576) for quantification of the primary transcript or TaqMan MicroRNA Assay (hsa-miR-576-3p) for quantification of miR-576-3p. Gene-specific primers were used to quantify SEC24B and GAPDH using the TaqMan system. For quantification of STING and IFN $\beta$ real-time qPCR was performed using gene-specific primers, iQ SYBR green supermix (Bio-Rad) according to the manufacturer's instructions, and the Bio-Rad CFX96 real-time PCR machine. The results were normalized to $\beta$-actin and GAPDH. Each PCR was performed in triplicate, and standard deviations were calculated. The amount of RNA is expressed in relative units with respect to mock treated samples.

Dual-luciferase assays. Targets of miR-576-3p were assessed by transfecting 293T cells with miR-576-3p mimic at a final concentration of $100 \mathrm{nM}$ and a 10:1 ratio of the pMIR-REPORT plasmid (gift of Eric Olson; UT Southwestern Medical Center) containing the $3^{\prime}$-UTR of the indicated gene and a Renilla reporter plasmid for normalization of transfection efficiency using Lipofectamine 2000 (Invitrogen). Cells were transfected with $50 \mathrm{ng}$ of pMIR-REPORT luciferase plasmid or plasmid containing a mutant $3^{\prime}$-UTR as a negative control. Mutated $3^{\prime}$-UTRs were obtained by deletion of the miR-576-3p microRNA-responsive element in the $3^{\prime}$-UTR by PCR mutagenesis. Firefly and renilla luciferase activity was measured using the Dual Luciferase Reporter assay system (Promega) in a luminometer (Fluoroskan Ascent; Thermo Fisher Scientific).

Poly (I:C) transfection. HBECs were reverse transfected for $72 \mathrm{~h}$ with miRNA control, or miR-576-3p mimic, or miR-576-3p inhibitor, or siRNAs to STING, P65 or IRF3 (Dharmacon), as indicated in the figure legends, at a concentration of $50 \mathrm{nM}$. Four to six hours before harvest, cells were transfected with Poly (I:C) (InvivoGen) at a final concentration of $1 \mu \mathrm{g} \mathrm{ml}^{-1}$ using Lipofectamine 2000 (Invitrogen), according to the manufacturer's instructions. As additional control, cells were transfected with Lipofectamine 2000 alone.

VSV infection and plaque assay. HBECs were transfected with control miRNA or with miR-576-3p mimic or inhibitor, or with siRNAs to STING, MAVS or TRAF3 (Dharmacon), as described above, and infected with VSV-GFP (Indiana strain) at MOI $0.1,1$ or 3 , as indicated in the figure legends. Infection time is described in the figure legends for each condition. Supernatants were collected and used for titration on BHK cells. Supernatants containing virus were serially diluted in serum-free DMEM and added to BHK cells. One hour after infection, complete DMEM containing $1 \%$ agarose was added to each well. GFP-positive plaques were counted $18-24 \mathrm{~h}$ after infection and used to calculate relative viral titres.
Assays with diverse viruses. HBECs were transfected in triplicate with miRNA mimics as described above. After $72 \mathrm{~h}$ of transfection, cells were infected with influenza virus (WSN strain), or vaccinia virus or HSV-1 at MOI of 5, 3 and 0.1 , respectively. Cell viabilities were measured after $18 \mathrm{~h}$ of infection with WSN and vaccinia virus and $96 \mathrm{~h}$ after HSV-1 infection using the CellTiter-Glo kit (Promega). Relative cell viabilities of miR-576-3p transfected cells were calculated relative to miRNA mimic control. As HBECs are resistant to infection, the same infection conditions were used for viral replication assays except that TPCK $\left(0.5 \mu \mathrm{g} \mathrm{ml}^{-1}\right)$ was added in the media during influenza virus and vaccinia virus infections. Supernatants were collected and plaque assays were performed in MDCK cells for influenza virus, in BHK cells for vaccinia virus and in Vero cells for HSV-1.

Conservation analysis of miR-576-3p and its targets. Multiple sequence alignment of pri-mir-576 among different species was performed using microRNAviewer (http://people.csail.mit.edu/akiezun/microRNAviewer/index.html ${ }^{29}$ The conservation of miR-576-3p target sites in $3^{\prime}$-UTR of STING, MAVS and TRAF3 were tested using TargetScan Release 6.2 (http://www.targetscan.org/).

\section{References}

1. Gottwein, E. \& Cullen, B. R. Viral and cellular microRNAs as determinants of viral pathogenesis and immunity. Cell Host Microbe 3, 375-387 (2008).

2. Santhakumar, D. et al. Combined agonist-antagonist genome-wide functional screening identifies broadly active antiviral microRNAs. Proc. Natl Acad. Sci. USA 107, 13830-13835 (2010).

3. Otsuka, M. et al. Hypersusceptibility to vesicular stomatitis virus infection in Dicer1-deficient mice is due to impaired miR24 and miR93 expression. Immunity 27, 123-134 (2007).

4. Lichty, B. D., Power, A. T., Stojdl, D. F. \& Bell, J. C. Vesicular stomatitis virus: re-inventing the bullet. Trends Mol. Med. 10, 210-216 (2004).

5. Barber, G. N. VSV-tumor selective replication and protein translation. Oncogene 24, 7710-7719 (2005).

6. Yoneyama, M. et al. The RNA helicase RIG-I has an essential function in double-stranded RNA-induced innate antiviral responses. Nat. Immunol. 5, 730-737 (2004).

7. Takeuchi, O. \& Akira, S. Recognition of viruses by innate immunity. Immunol. Rev. 220, 214-224 (2007).

8. Kawai, T. et al. IPS-1, an adaptor triggering RIG-I- and Mda5-mediated type I interferon induction. Nat. Immunol. 6, 981-988 (2005)

9. Meylan, E. et al. Cardif is an adaptor protein in the RIG-I antiviral pathway and is targeted by hepatitis C virus. Nature 437, 1167-1172 (2005).

10. Seth, R. B., Sun, L., Ea, C. K. \& Chen, Z. J. Identification and characterization of MAVS, a mitochondrial antiviral signaling protein that activates NF-kappaB and IRF 3. Cell 122, 669-682 (2005).

11. $\mathrm{Xu}, \mathrm{L}$. G. et al. VISA is an adapter protein required for virus-triggered IFN-beta signaling. Mol. Cell 19, 727-740 (2005).

12. Ishikawa, H. \& Barber, G. N. STING is an endoplasmic reticulum adaptor that facilitates innate immune signalling. Nature 455, 674-678 (2008).

13. Ishikawa, H., Ma, Z. \& Barber, G. N. STING regulates intracellular DNAmediated, type I interferon-dependent innate immunity. Nature 461, 788-792 (2009).

14. Sun, L., Wu, J., Du, F., Chen, X. \& Chen, Z. J. Cyclic GMP-AMP synthase is a cytosolic DNA sensor that activates the type I interferon pathway. Science 339, 786-791 (2013)

15. Wu, J. et al. Cyclic GMP-AMP is an endogenous second messenger in innate immune signaling by cytosolic DNA. Science 339, 826-830 (2013).

16. Ramirez, R. D. et al. Immortalization of human bronchial epithelial cells in the absence of viral oncoproteins. Cancer Res. 64, 9027-9034 (2004)

17. Hu, G. et al. MicroRNA-98 and let-7 confer cholangiocyte expression of cytokine-inducible Src homology 2-containing protein in response to microbial challenge. J. Immunol. 183, 1617-1624 (2009).

18. Tili, E. et al. Modulation of miR-155 and miR-125b levels following lipopolysaccharide/TNF-alpha stimulation and their possible roles in regulating the response to endotoxin shock. J. Immunol. 179, 5082-5089 (2007).

19. Wang, D. et al. MiR-125b reduces porcine reproductive and respiratory syndrome virus replication by negatively regulating the NF-kappaB pathway. PLoS ONE 8, e55838 (2013).

20. Lewis, B. P., Burge, C. B. \& Bartel, D. P. Conserved seed pairing, often flanked by adenosines, indicates that thousands of human genes are microRNA targets. Cell 120, 15-20 (2005).

21. Kato, H. et al. Length-dependent recognition of double-stranded ribonucleic acids by retinoic acid-inducible gene-I and melanoma differentiation-associated gene 5. J. Exp. Med. 205, 1601-1610 (2008).

22. Barrett, T. et al. NCBI GEO: archive for functional genomics data sets--update. Nucleic Acids Res. 41, D991-D995 (2013)

23. Zhu, S. et al. The microRNA miR-23b suppresses IL-17-associated autoimmune inflammation by targeting TAB2, TAB3 and IKK-alpha. Nat. Med. 18, 1077-1086 (2012). 
24. Taganov, K. D., Boldin, M. P., Chang, K. J. \& Baltimore, D. NF-kappaBdependent induction of microRNA miR-146, an inhibitor targeted to signaling proteins of innate immune responses. Proc. Natl Acad. Sci. USA 103, 12481-12486 (2006).

25. Balachandran, S. \& Barber, G. N. Defective translational control facilitates vesicular stomatitis virus oncolysis. Cancer Cell 5, 51-65 (2004).

26. Wu, Z., Liu, D. \& Sui, Y. Quantitative assessment of hit detection and confirmation in single and duplicate high-throughput screenings. J. Biomol. Screen. 13, 159-167 (2008).

27. Subramanian, A. et al. Gene set enrichment analysis: a knowledge-based approach for interpreting genome-wide expression profiles. Proc. Natl Acad. Sci. USA 102, 15545-15550 (2005).

28. Mata, M. A. et al. Chemical inhibition of RNA viruses reveals REDD1 as host defense factor. Nat. Chem. Biol. 7, 712-719 (2011).

29. Kiezun, A. et al. miRviewer: a multispecies microRNA homologous viewer. BMC Res. Notes 5, 92 (2012).

\section{Acknowledgements}

We thank J. Yang for technical assistance; S. Wei, G. Henry and M. Vishwanath for assistance in the high-throughput screening facility; $\mathrm{C}$. Bu for assistance with statistical analysis; E. Olson and A. Garcia-Sastre for reagents; D. Levy for helpful suggestions and critical reading of the manuscript; and M. Potts for help with the miRNA mimic library.
We thank Angela Diehl for outstanding illustration. Funding was provided by NIH R01 GM067159, R01 AI079110, R01 AI089539, CPRIT RP121003-RP120718-P2, T32-CA124334 and F32-AI091409.

\section{Author contributions}

M.L.Y., K.Z., G.N.B., B.A.P., M.A.W. and B.M.A.F. conceived and designed the experiments. M.L.Y. and K.Z. performed the experiments. M.L.Y., K.Z., R.S., C.V.F., G.N.B., B.A.P., M.A.W. and B.M.A.F. analysed the data. R.S., C.V.F., G.N.B., B.A.P., M.A.W. and B.M.A.F. contributed the reagents/materials/analysis tools. M.L.Y. and B.M.A.F. wrote the paper.

\section{Additional information}

Supplementary Information accompanies this paper at http://www.nature.com/ naturecommunications

Competing financial interests: The authors declare no competing financial interests.

Reprints and permission information is available online at http://npg.nature.com/ reprintsandpermissions/

How to cite this article: Yarbrough, M. L. et al. Primate-specific miR-576-3p sets host defense signalling threshold. Nat. Commun. 5:4963 doi: 10.1038/ncomms5963 (2014). 